\title{
La angiotomografía coronaria no fue superior a los estudios funcionales en pacientes con enfermedad coronaria crónica estable
}

\author{
Angiotomography was not superior to functional studies in patients with stable coronary heart disease
}

\section{Objetivos}

Comparar la efectividad de dos estrategias de estudio inicial en pacientes con sospecha de enfermedad coronaria (EC) que a juicio de los médicos tratantes no requirieran estudios invasivos (pacientes con enfermedad crónica estable): evaluación inicial de la anatomía coronaria con angiotomografía coronaria multicorte vs. evaluación funcional. Como objetivos secundarios: comparar los costos de ambos métodos diagnósticos y la radiación que ambos generarían en los pacientes.

\section{Diseño, lugar y pacientes}

El estudio PROMISE fue un estudio prospectivo, aleatorizado y multicéntrico. Se incluyeron 10.003 pacientes (edad media: 60,8 \pm 8,3 años) de 193 centros de Canadá y Estados Unidos, la mayoría con dolor precordial estable, de reciente instalación, sin antecedentes conocidos de EC.

\section{Asignación de pacientes e intervención}

Se los asignó de manera aleatoria a una estrategia de estudio anatómico con angiotomografía coronaria multicorte (TCMS) vs. estudios funcionales (ergometría, ecocardiograma estrés o SPECT).

Tabla 1. Resultados primarios y secundarios acorde a cada grupo de estudio.

\begin{tabular}{|c|c|c|c|c|}
\hline Resultado & $\begin{array}{c}\text { TCMS } \\
(\mathrm{N}=4.996)\end{array}$ & $\begin{array}{l}\text { Test funcional } \\
(\mathrm{N}=5.006)\end{array}$ & HR (IC $95 \%)$ & P valo \\
\hline Resultado primario & 164 & 151 & $1,04(0,83$ a 1,29$)$ & 0,75 \\
\hline Muerte por cualquier causa & 74 & 75 & & \\
\hline Infarto de miocardio no fatal & 30 & 40 & & \\
\hline Internación por angina inestable & 61 & 41 & & \\
\hline Complicación mayor periprocedimiento & 4 & 5 & & \\
\hline Resultado primario más cateterismo sin evidencia de enfermedad coronaria & 332 & 353 & $0,91(0,78$ a 1,06$)$ & 0,22 \\
\hline Muerte 0 infarto no fatal & 104 & 112 & $0,88(0,67$ a 1,15$)$ & 0,35 \\
\hline Muerte, infarto no fatal o internación por angina & 162 & 148 & $1,04(0,84$ a 1,31$)$ & 0,70 \\
\hline
\end{tabular}

TCMS: angiotomografía computada multicorte. HR: Hazard ratio. IC 95\%: intervalo de confianza del $95 \%$.

En el grupo TCMS fue mayor la cantidad de pacientes sometidos a cinecoronariografía ( $12,2 \%$ vs. $8,1 \%)$ y a procedimientos de revascularización dentro de los 90 días: $6,2 \%$ vs. $3,2 \%(p<0,001)$. La exposición media a radiación por paciente que recibió radiación fue menor en el grupo de TCMS con respecto a estudio funcional $(10,0 \mathrm{mSv}$ vs $11,3 \mathrm{mSv})$, pero el $32,6 \%$ de los pacientes en la rama funcional no tuvo exposición a radiación, por ende la exposición global fue mayor en el grupo TCMS (media $12,0 \mathrm{mSv}$ vs. $10,1 \mathrm{mSv} ; \mathrm{P}<\mathrm{a} 0,001$ ). En términos de costos netos, la tomografía fue $\$ 279$ dólares ser más costosa a los 90 días a expensas de mayor necesidad de revascularización en dicha

\section{Comentario}

Es importante remarcar que a pesar que no hubo diferencias en el punto final primario entre ambas ramas, la decisión de indicar un estudio $u$ otro debe ser hecha paciente por paciente, conociendo las ventajas y limitaciones de cada método en términos de sensibilidad y especificidad diagnóstica. Por ejemplo, la angiotomografía coronaria multicorte posee un alto valor predictivo negativo en el paciente con probabilidad pretest baja a intermedia siendo una herramienta de gran valor en las centrales de emergencias para descartar rápidamente síndromes coronarios agudos ${ }^{1}$. Por otro lado, existen grupos en los cuales la capacidad diagnóstica del método cae notablemente como por ejemplo efectuar angiotomografía coronaria multicorte en pacientes añosos donde sabemos que la mayor prevalencia de cal-
PROMISE Research Group. N Engl J Med 2015;372:1291-1300. Medición de resultados principales

Los resultados del estudio fueron dados en tiempo real y el equipo tratante tomó las decisiones ulteriores. Los pacientes fueron seguidos por 25 meses (rango inter-cuartilo de 18 a 34 meses). Se definió como resultado primario a la incidencia de muerte, infarto agudo de miocardio, hospitalización por angina inestable y/o complicaciones mayores del procedimiento (accidente cerebrovascular, hemorragia mayor, anafilaxia y/o insuficiencia renal). El $87,7 \%$ de los pacientes tenía dolor precordial o disnea de esfuerzo y la probabilidad pre-test de EC era de $53,3 \pm 21.4 \%$. De los 4.996 pacientes asignados a la estrategia funcional, se les realizó SPECT al $67,3 \%$, ecocardiograma estrés al $22,5 \%$ y ergometría al 10,2\%. El análisis se efectuó por intención de tratar.

\section{Resultados principales}

El resultado primario ocurrió en el 3,3\% de los pacientes del grupo TCMS y en el $3 \%$ de los pacientes con prueba funcional. No se observaron diferencias en la incidencia del resultado primario en ambos grupos (ver Tabla 1). rama. A los dos años de seguimiento, la diferencia con respecto a estudios funcionales fue tan sólo de $\$ 30$ dólares.

\section{Conclusiones}

En conclusión, la evaluación inicial de la anatomía coronaria con TCMS no mostró beneficios respecto de la realización de pruebas funcionales en pacientes con síntomas sugestivos de enfermedad coronaria que no requirieran evaluación invasiva.

Fuente de financiamiento: Instituto Nacional de Corazón, Pulmón y Sangre (NHLBI). No se reportan conflictos de interés.

Francisco José Romeo [ Senvicio de Hemodinamia y Cardiologia Intervencionista del Hospital Italiano de Buenos Aires. Francisco.romeo@ hospitalitaliano.org.ar ] Romeo F. La angiotomografía coronaria no fue superior a los estudios funcionales en pacientes con enfermedad coronaria crónica estable. Evid Act Pract Ambul. 2017;20(1):5. Comentado de: Douglas PS, y col. Outcomes of Anatomical versus Functional Testing for Coronary Artery Disease. N Engl J Med 2015;372:1291-1300. PMID: 25773919.

1. Budoff MJ, y col. Diagnostic performance of 64-multidetector row coronary computed tomographic angiography for evaluation of coronary artery stenosis in individuals without known coronary artery disease: Results from the prospective multicenter accuracy (assessment by coronary computed tomographic angiography of individuals undergoing invasive coronary angiography) trial. Journal of the American College of Cardiology. 2008:52:1724-1732.

2. Zhang S, y col. Accuracy of mdct in assessing the degree of stenosis caused by calcified coronary artery plaques. AJR. American journal of roentgenology. $2008 ; 191: 1676-1683$. 3. Khan A, y col. Comparison of radiation dose and image quality: 320-mdct versus 64-mdct coronary angiography. AJR. American journal of roentgenology. 2011;197:163-168. 